\section{International Scientific Journal Theoretical \& Applied Science}

p-ISSN: 2308-4944 (print)

e-ISSN: 2409-0085 (online)

Year: 2015

Issue: 04

Volume: 24

Published: $30.04 .2015 \quad$ http://T-Science.org

SECTION 2. Applied mathematics. Mathematical modeling.
Alexandr Nikolayevich Shevtsov candidate of technical sciences, member of PILA (USA),

Department of «Mathematics»,

Taraz state University M.Kh. Dulati, Kazakhstan Shev_AlexXXXX@mail.ru

Aigul Izdibaevna Chanbaeva senior lecturer, Department of «Mathematics», Taraz state University M.Kh. Dulati, Kazakhstan

Sara Ashimovna Suleymenova teacher of mathematics

Taraz College of engineering and business,

\title{
ABOUT APPROXIMATE CALCULATION OF DEFINITE INTEGRAL
}

Abstract: The paper discusses some algorithms, methods and techniques for custom computing definite integrals.

Key words: integral, numerical method, approximation of the solution.

Language: Russian

Citation: Shevtsov AN, Chanbaeva AI, Suleymenova SA (2015) ABOUT APPROXIMATE CALCULATION OF DEFINITE INTEGRAL. ISJ Theoretical \& Applied Science 04 (24): 281-286.

Soi: http://s-o-i.org/1.1/TAS*04(24)51 Doi: croskef http://dx.doi.org/10.15863/TAS.2015.04.24.51

\section{ОБ ОДНОМ ПРИБЛИЖЕННОМ ВЫЧИСЛЕНИИ ОПРЕДЕЛЕННОГО ИНТЕГРАЛА}

Аннотация: В работе рассмотрены некоторые алгоритмы, методы и способы для вычисления нестандартных определенных интегралов.

Ключевые слова: интеграл, численный метод, аппроксимация решения.

Рассмотрим интеграл и методы его аппроксимации известными функциями.

$$
\begin{aligned}
& \int_{0}^{1} \sqrt{\frac{1-t}{1+t}} d t \\
& \int_{0}^{1} \sqrt{\frac{1-r^{2}}{1+r^{2}}} d r
\end{aligned}
$$

Очевидно, что аналитическое решение неопределенного интеграла на Maple будет следующим:

restart:
$y:=\operatorname{sqrt}\left(\frac{\left(1-x^{2}\right)}{\left(1+x^{2}\right)}\right):$
$\operatorname{int}(y, x)$

$$
\frac{\sqrt{-\frac{x^{2}-1}{x^{2}+1}\left(x^{2}+1\right)^{3 / 2} \sqrt{-x^{2}+1}(2 \operatorname{EllipticF}(x, \mathrm{I})-\operatorname{EllipticE}(x, \mathrm{I}))}}{\sqrt{-\left(x^{2}+1\right)\left(x^{2}-1\right)} \sqrt{-x^{4}+1}}
$$

Причем при нахождении определенного интеграла получим

$$
2 \text { EllipticK(I) - EllipticE(I) }
$$

а само численное значение равно

$$
0.711958659+0 . I
$$

Также несложно проверить, что в случае (1)

$$
\int_{0}^{1} r \sqrt{\frac{1-r^{2}}{1+r^{2}}} d r
$$

интеграл легко сводится к табличному:

$$
\begin{aligned}
& \text { restart: } \\
& y:=\operatorname{sqrt}\left(\frac{\left(1-r^{2}\right)}{\left(1+r^{2}\right)}\right): \\
& \operatorname{Int}(\mathrm{r} \cdot y, r)=\operatorname{int}(\mathrm{r} \cdot y, r) ; \\
& \operatorname{evalf}(\operatorname{int}(\mathrm{r} \cdot y, \mathrm{r}=0 . .1)) ;
\end{aligned}
$$




$$
\int r \sqrt{\frac{-r^{2}+1}{r^{2}+1}} \mathrm{~d} r=\frac{1}{2} \frac{\sqrt{-\frac{r^{2}-1}{r^{2}+1}\left(r^{2}+1\right)\left(\sqrt{-r^{4}+1}+\arcsin \left(r^{2}\right)\right)}}{\begin{array}{c}
\sqrt{-\left(r^{2}-1\right)\left(r^{2}+1\right)} \\
0.2853981635
\end{array}}
$$

В случае же с (2), мы имеем дело с подъинтегральной функцией

$$
y=\sqrt{\frac{1-r^{2}}{1+r^{2}}}
$$
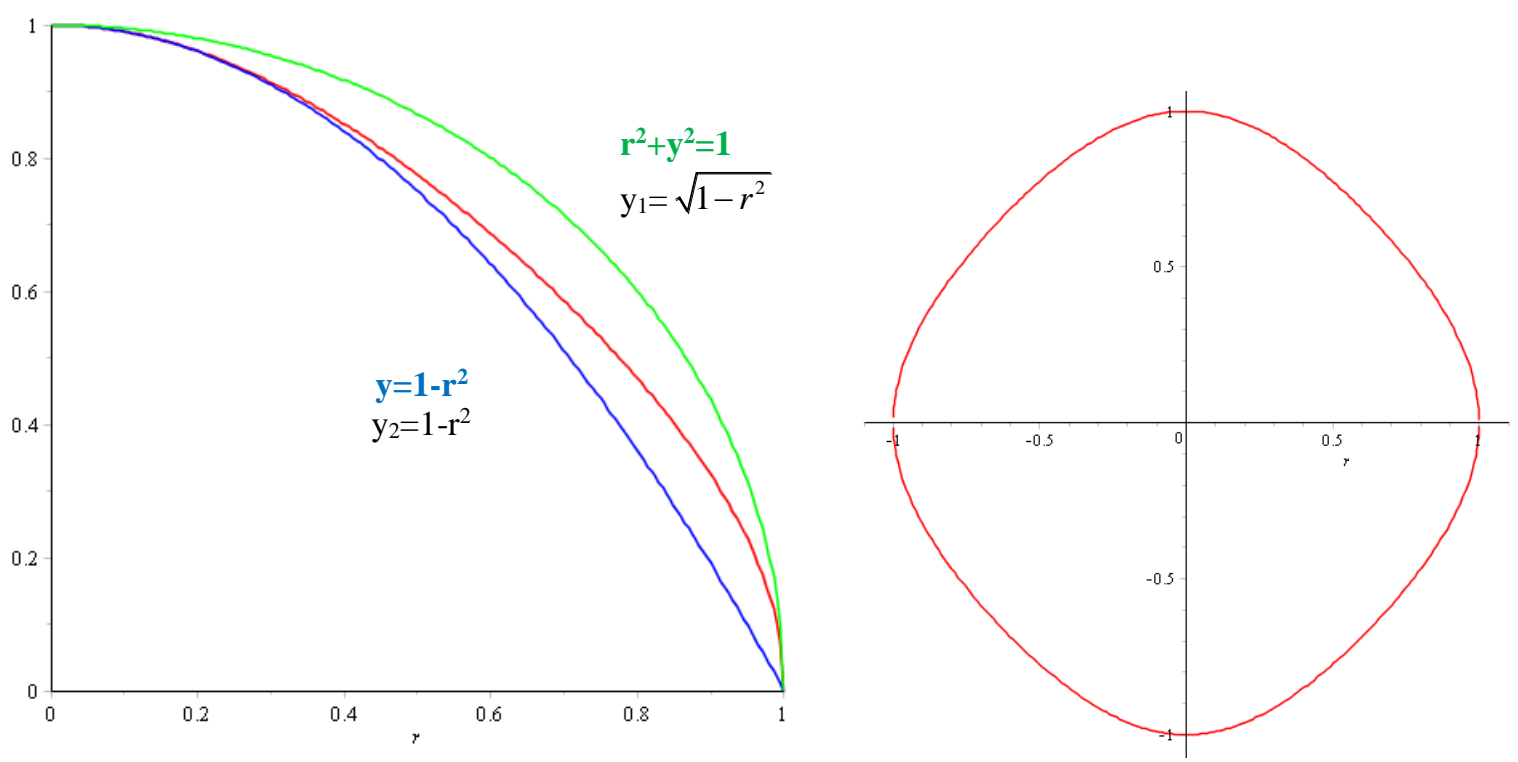

Рисунок 1 - Аппроксимация и график функции при $t=r^{2}$.

$$
\begin{aligned}
y l & :=\operatorname{sqrt}\left(1-r^{2}\right): \\
y 2 & :=1-r^{2}: \\
z & :=\frac{1}{e} \cdot \operatorname{int}(y 1, r)+\frac{(e-1)}{\mathrm{e}} \cdot \operatorname{int}(y 2, r) ; \\
z l & :=\frac{1}{e} \cdot \operatorname{int}(y 1, r=0 \ldots 1)+\frac{(e-1)}{\mathrm{e}} \cdot \operatorname{int}(y 2, r=0 \ldots 1) ; \\
e & :=2.71828182845904523536: \\
\text { evalf }(z 1) ; \quad & \frac{1}{2} r \sqrt{-r^{2}+1}+\frac{1}{2} \arcsin (r) \\
\int R \mathrm{~d} r & =\frac{(e-1)\left(-\frac{1}{3} r^{3}+r\right)}{e} \\
\int_{0}^{1} R \mathrm{~d} r=\frac{1}{4} \frac{\pi}{e}+\frac{2}{3} \frac{e-1}{e} & 0.7103455434
\end{aligned}
$$

В результате имеем 


$$
\sqrt{\frac{1-r^{2}}{1+r^{2}}} \rightarrow \frac{\frac{1}{2} r \sqrt{1-r^{2}}+\frac{1}{2} \arcsin (r)+(e-1) r\left(1-\frac{1}{3} r^{2}\right)}{e}
$$

Аналогично для (1) получим:

$$
\begin{gathered}
\int \sqrt{\frac{1-r}{1+r}} \mathrm{~d} r=\frac{\sqrt{-\frac{r-1}{1+r}}(1+r)\left(\sqrt{-r^{2}+1}+\arcsin (r)\right)}{\sqrt{-(1+r)(r-1)}} \\
\int_{0}^{1} \sqrt{\frac{1-r}{1+r}} \mathrm{~d} r=0.570796327 \\
\int R \mathrm{~d} r=-\frac{2}{3} \frac{(1-r)^{3 / 2}}{e}+\frac{(e-1)\left(r-\frac{1}{2} r^{2}\right)}{e} \\
\int_{0}^{1} R \mathrm{~d} r=\frac{2}{3 e}+\frac{1}{2} \frac{e-1}{e} \\
0.5613132402
\end{gathered}
$$

Аналогично для $t=r^{3}$ получим:

$$
\begin{aligned}
& \int \sqrt{\frac{-r^{3}+1}{r^{3}+1}} d r=\int \sqrt{\frac{-r^{3}+1}{r^{3}+1}} d r \\
& \int_{0}^{1} \sqrt{\frac{-r^{3}+1}{r^{3}+1}} \mathrm{~d} r=0.7831403974
\end{aligned}
$$
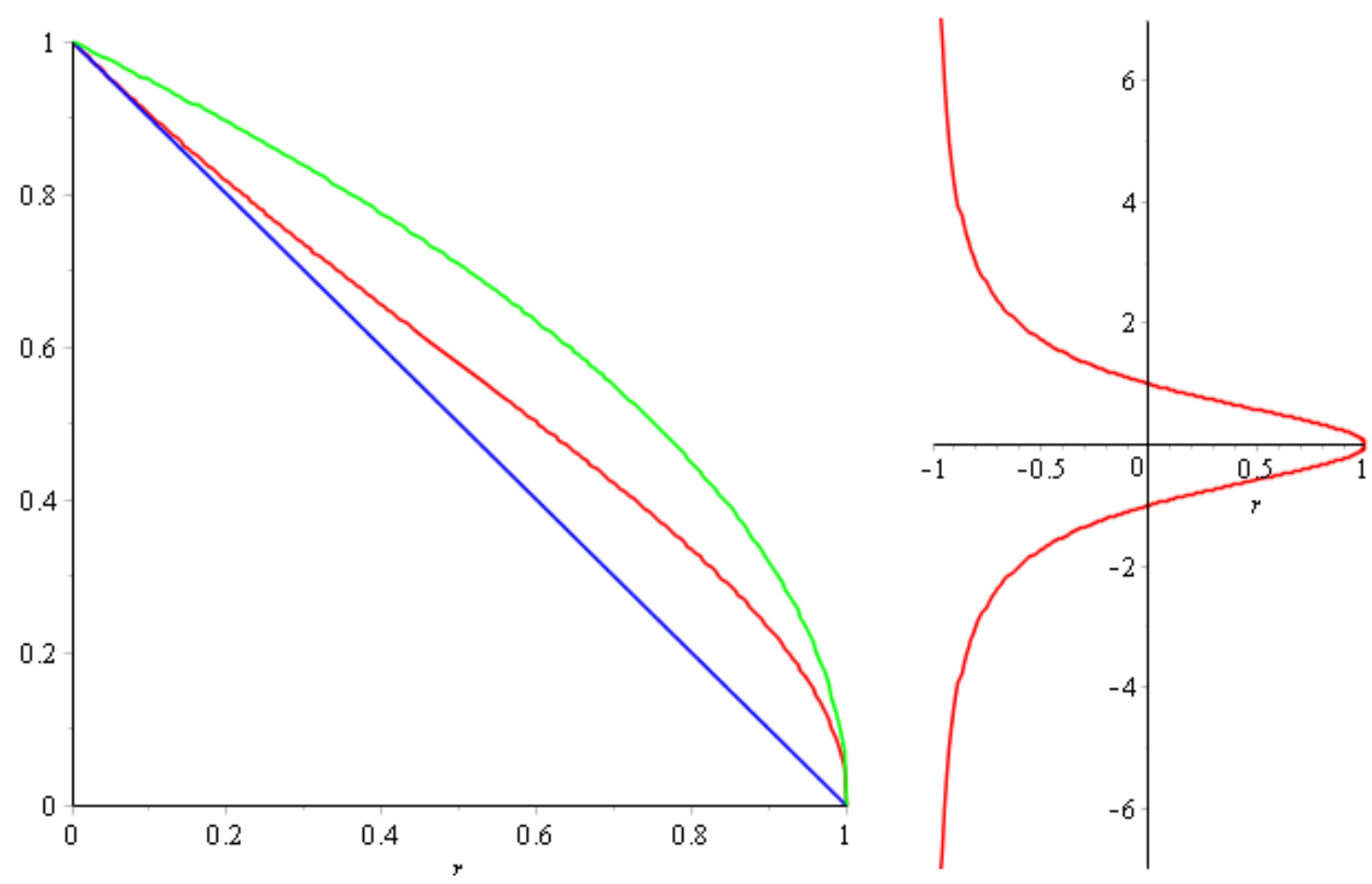

Рисунок 2 - Аппроксимация и график функции при $t=r$. 


$$
\begin{aligned}
& \int R \mathrm{~d} r=\frac{1}{e}\left(\frac{2}{5} r \sqrt{-r^{3}+1}\right. \\
& -\frac{1}{\sqrt{-r^{3}+1}}\left(\frac { 2 } { 5 } \mathrm { I } \sqrt { 3 } \sqrt { \mathrm { I } ( r + \frac { 1 } { 2 } - \frac { 1 } { 2 } \mathrm { I } \sqrt { 3 } ) \sqrt { 3 } } \sqrt { \frac { r - 1 } { - \frac { 3 } { 2 } + \frac { 1 } { 2 } \mathrm { I } \sqrt { 3 } } } \sqrt { - \mathrm { I } ( r + \frac { 1 } { 2 } + \frac { 1 } { 2 } \mathrm { I } \sqrt { 3 } ) \sqrt { 3 } } \text { EllipticF } \left(\frac{1}{3} \sqrt{3}\right.\right. \\
& \left.\left.\left.\quad \sqrt{\mathrm{I}\left(r+\frac{1}{2}-\frac{1}{2} \mathrm{I} \sqrt{3}\right) \sqrt{3}}, \sqrt{\frac{\mathrm{I} \sqrt{3}}{-\frac{3}{2}+\frac{1}{2} \mathrm{I} \sqrt{3}}}\right)\right)\right)+\frac{(e-1)\left(-\frac{1}{4} r^{4}+r\right)}{e} \\
& \int_{0}^{1} R \mathrm{~d} r=\frac{1}{3} \frac{\mathrm{B}\left(\frac{1}{3}, \frac{3}{2}\right)}{e}+\frac{3}{4} \frac{e-1}{e}
\end{aligned}
$$
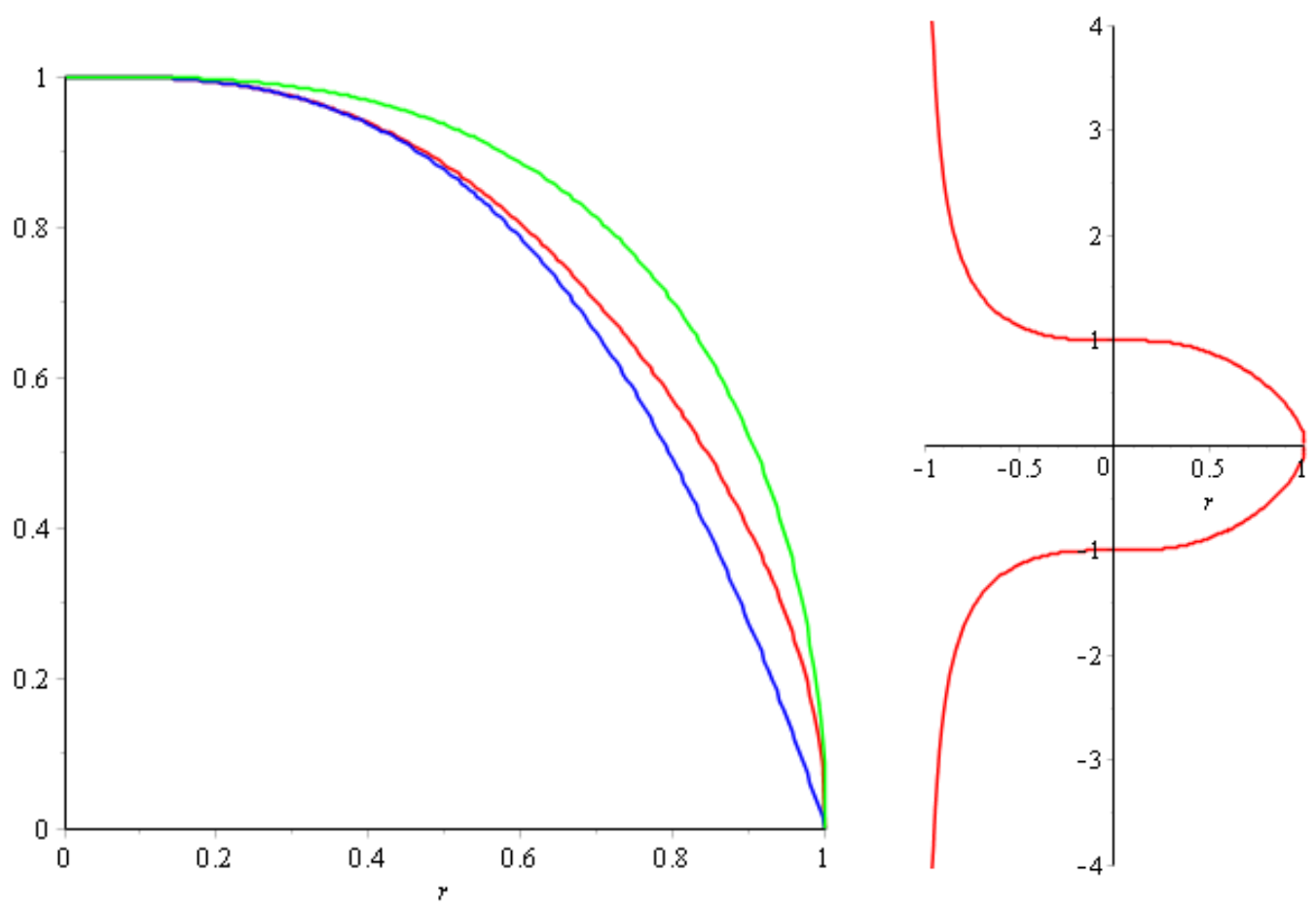

Рисунок 3 - Аппроксимация и график функции при $t=r^{3}$.

Аналогичная ситуация повторяется и для более высоких порядков. Точность расчета функции $R$ с ростом степени возрастает на порядок. 

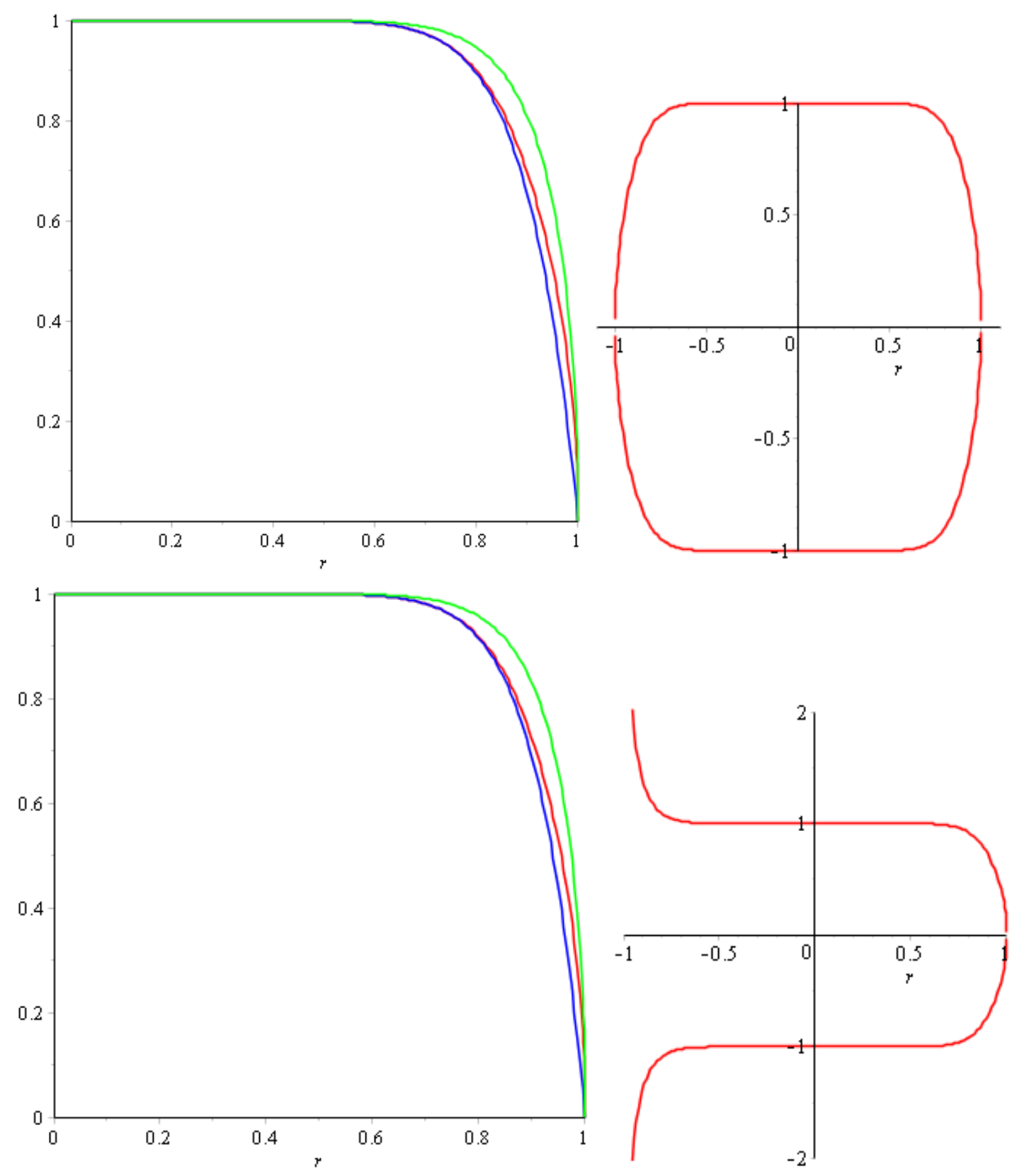

Рисунок 4 - Аппроксимация и график функции при $t=r^{10}, \quad t=r^{11}$.

Полученные формулы $R^{*}=\int_{0}^{1} R d r$ запишем в таблицу:

\begin{tabular}{|l|c|}
\hline$n$, & $R^{*}, \quad R^{*}=\int_{0}^{1} R d r$ \\
\hline $1=r^{n}$ & $\int_{0}^{1} R \mathrm{~d} r=\frac{2}{3 e}+\frac{1}{2} \frac{e-1}{e}$ \\
\hline
\end{tabular}

\begin{tabular}{|l|l|}
\hline 2 & $\int_{0}^{1} R \mathrm{~d} r=\frac{1}{4} \frac{\pi}{e}+\frac{2}{3} \frac{e-1}{e}$ \\
\hline 3 & $\int_{0}^{1} R \mathrm{~d} r=\frac{1}{3} \frac{\mathrm{B}\left(\frac{1}{3}, \frac{3}{2}\right)}{e}+\frac{3}{4} \frac{e-1}{e}$ \\
\hline 4 & $\int_{0}^{1} R \mathrm{~d} r=\frac{1}{4} \frac{\mathrm{B}\left(\frac{1}{4}, \frac{3}{2}\right)}{e}+\frac{4}{5} \frac{e-1}{e}$ \\
\hline
\end{tabular}

ISPC The Combination of Technology \& Education, Östersund, Sweden 


\begin{tabular}{|l|l}
\hline 5 & $\int_{0}^{1} R \mathrm{~d} r=\frac{1}{5} \frac{\mathrm{B}\left(\frac{1}{5}, \frac{3}{2}\right)}{e}+\frac{5}{6} \frac{e-1}{e}$ \\
\hline 6 & $\int_{0}^{1} R \mathrm{~d} r=\frac{1}{6} \frac{\mathrm{B}\left(\frac{1}{6}, \frac{3}{2}\right)}{e}+\frac{6}{7} \frac{e-1}{e}$ \\
\hline 7 & $\int_{0}^{1} R \mathrm{~d} r=\frac{1}{7} \frac{\mathrm{B}\left(\frac{1}{7}, \frac{3}{2}\right)}{e}+\frac{7}{8} \frac{e-1}{e}$ \\
\hline 8 & $\int_{0}^{1} R \mathrm{~d} r=\frac{1}{8} \frac{\mathrm{B}\left(\frac{1}{8}, \frac{3}{2}\right)}{e}+\frac{8}{9} \frac{e-1}{e}$ \\
\hline
\end{tabular}

\begin{tabular}{|l|l|}
\hline 9 & $\int_{0}^{1} R \mathrm{~d} r=\frac{1}{9} \frac{\mathrm{B}\left(\frac{1}{9}, \frac{3}{2}\right)}{e}+\frac{9}{10} \frac{e-1}{e}$ \\
\hline 10 & $\int_{0}^{1} R \mathrm{~d} r=\frac{1}{10} \frac{\mathrm{B}\left(\frac{1}{10}, \frac{3}{2}\right)}{e}+\frac{10}{11} \frac{e-1}{e}$ \\
\hline
\end{tabular}

Из полученных результатов построить обшую формулу для интеграла (1):

$$
\int_{0}^{1} \sqrt{\frac{1-t^{n}}{1+t^{n}}} d t=\frac{1}{e}\left(\frac{1}{n} B\left(\frac{1}{n}, \frac{3}{2}\right)+\frac{n}{n+1}(e-1)\right) .
$$

\section{References:}

1. Arkhipov, Sadovnichiy, Chubarikov (1999) Lektsii po matematicheskomu analizu. Uchebnik.analiz. 1999. -635 p.

2. Aksenov (2000) Matematicheskiy analiz. (Integraly, zavisyashchie ot parametra. Dvoynye integraly. Krivolineynye integraly.) Uchebnoe posobie $\mathrm{SPb}$. 2000. -145 p.

3. Stefan Banakh (1966) Differentsial'noe i integral'noe ischislenie. 1966. -437 p.

4. Budak BM, Fomin SV (1965) Kratnye integoaly i ryady. Uchebnik.1965. -606 p.

5. Kochetkov (1999) Kratkiy kurs matematicheskogo analiza, lineynoy algebry i matematicheskog modelirvaniya. MGII. 1999. $60 \mathrm{p}$.

6. Zorich VA (1997) Matematicheskiy analiz. V 2-kh chastyakh. Uchebnik. 1- 1997, 2 - 1984. $567+640 \mathrm{p}$.
7. Il'in, Poznyak (2005) Osnovy matematicheskogo analiza. 2002-2005gody. V 2-kh chastyakh. 1 ch. 644 p., 2 ch. 464 p.

8. Tikhomandridskiy (1895) Teoriya ellipticheskikh" integralov". 133 dvoynykh str. Raritetnoe izdanie. Khar'kov.

9. LEONARD ZYLER (1956) INTEGRAL"NOE ISChISLENIE. V 3-kh tomakh. PEREVOD S LATINSKOGO. 1956-1958.

10. Butuzov VF, Krutitskaya NC, Medvedev GN, Shishkin AA (2002) Matematicheskiy analiz v voprosakh i zadachakh: Ucheb. posobie. 5-e izd., ispr. 2002. $480 \mathrm{p}$.

11. Lungu, Makarov (2005) Vysshaya matematika. Rukovodstvo k resheniyu zadach. Chast' 1 . 2005. -315 p. 